\title{
Dynamical characterization of rotationally hindered species in liquids
}

\author{
A. Medina, ${ }^{\text {a) }}$ J. M. M. Roco, A. Calvo Hernández, and S. Velasco \\ Departamento de Física Aplicada, Facultad de Ciencias, Universidad de Salamanca, \\ 37008 Salamanca, Spain
}

(Received 28 July 2005; accepted 17 October 2005)

\begin{abstract}
The rotational dynamics of $\mathrm{HCl}$ in liquid $\mathrm{Ar}$ has been studied by means of molecular-dynamics simulations. We calculate the lifetimes of weakly bound $\mathrm{HCl}-\mathrm{Ar}$ dimers induced by the anisotropic pair interaction. It is shown that, although lifetimes are small with respect to the reorientational decorrelation, the time interval between the breaking down and formation of the next dimer is negligibly small. Thus, with respect to the rotational dynamics of the probe, the effect is similar to that and eventually would cause a time-stable complex. This provokes a peculiar hindered rotation of the diatomic in the liquid which is macroscopically embodied in the infrared spectrum of the solution as a $Q$-branch nonexistent otherwise. (C) 2005 American Institute of Physics.
\end{abstract}

[DOI: $10.1063 / 1.2137699]$

\section{INTRODUCTION}

This work constitutes a step forward in a series of papers involved with three entangled problems: the $Q$-branch absorption that the experimental infrared spectra of some diatomic highly diluted in simple liquids show, anisotropic solute-solvent interaction potentials in dense fluids, and the eventual translation to liquid solvent densities of the van der Waals complexes well known in the gas phase.

The existence of an intense $Q$-branch absorption in the infrared spectra of some diatomics highly diluted in simple liquids was shown experimentally many years ago. ${ }^{1}$ This $Q$ branch is located close to the fundamental vibration frequency of the diatomic and it is dipole forbidden for the isolated molecule. Although for some systems its intensity is even larger than those of the $P$ and $R$ branches, ${ }^{2,3}$ its physical interpretation was an open question for many years. ${ }^{4,5} \mathrm{Re}$ cently, classical molecular-dynamics (MD) simulations have proven to be a powerful tool to look into the physical origin of such absorption. Particularly for $\mathrm{HCl}$ solved in liquid $\mathrm{Ar}$ or other simple liquid mixtures, simulated spectra recovered the main structure and relative intensities of the experimental measures. ${ }^{6}$ This was possible by introducing in the simulations realistic anisotropic solute-solvent potentials that in the low-density limit reproduce van der Waals complexation. ${ }^{7}$

In more general terms, a challenging macroscopic manifestation of the peculiarities of diatom-atom interactions was found: the $P-R$ interbranch region of the infrared spectra shows a particular sensitivity to the anisotropy of the potential. Different anisotropic potentials, for instance, for the long-time studied pair $\mathrm{HCl}-\mathrm{Ar}$, yielded to very different absorption bands. ${ }^{8}$ This suggests that this interbranch region could be considered as a severe test for the validity of anisotropic interaction surfaces at liquid solvent densities. Moreover, simulations were capable of reproducing the main types of vibration-rotation spectra experimentally observed for dif-

\footnotetext{
${ }^{a)}$ Author to whom correspondence should be addressed; electronic mail: amd385@usal.es
}

ferent systems and different liquid conditions by taking a prototypical system at fixed thermodynamical conditions and changing the anisotropy of the molecular probe-solvent interactions. ${ }^{9}$ In particular, an isotropic interaction surface, that in terms of rotational dynamics reproduces a free rigidrotor evolution, yields to a doublet structure with wellseparated $P$ and $R$ branches and a deep well in the interbranch region. ${ }^{10}$ On the other extreme, a unique collapsed branch is reproduced through an anisotropic potential provoking a slow monotonic decorrelation of the dipole moment of the diatomic, characteristic of a rotational diffusion process.

In the case of spectra showing a $Q$ branch, structural studies $^{8}$ revealed a privileged presence of solvent atoms at a distance and orientation with respect to the internuclear axis of the diatomic that resembles the geometry of the van der Waals complexes. Thus, it appears that some kind of weakly bound complexation directly affects the rotational evolution of the diatomic, although the precise meaning of this complexation should be investigated. Speaking in terms of rotational dynamics, it was found that a characteristic hindered rotor evolution far away from the usual limits: quasi-free rigid rotor for low gas densities and rotational diffusion for high-density fluids. ${ }^{11}$ One of the main objectives of this paper is to extend previous works to perform a dynamical characterization of weakly bound $\mathrm{HCl}-\mathrm{Ar}$ dimers at liquid solvent densities by directly studying their formationdissociation process. Similar techniques to those usually employed in computer simulation works to study, for instance, hydrogen bonding in liquids ${ }^{12,13}$ will be developed.

The structure of the paper is as follows. Section II is devoted to detailing the simulations we have developed and briefly the interaction potentials we employ. In Sec. III we explain three different definitions we assume for dimerization. With these criteria we calculate (Sec. IV) the lifetimes of $\mathrm{HCl}-\mathrm{Ar}$ weak conformations in liquid and also the time percentage this complexation exists. Section V contains an analysis of two different time autocorrelation functions that 
allow us to characterize the reorientational dynamics of the molecular probe in the liquid. Finally, in Sec. VI, we summarize the main conclusions of the work.

\section{DETAILS OF POTENTIALS AND SIMULATIONS}

We elect the system $\mathrm{HCl}-\mathrm{Ar}$ as an archetype for simple molecule-atom pairs interacting through a highly anisotropic potential that in the low-density limit give rise to a van der Waals dimer. ${ }^{14}$ In the liquid phase this prototype is specially interesting due to several reasons. First, at several thermodynamic conditions its infrared spectrum lacks of fine rotational structure, ${ }^{15}$ and thus it admits a semiclassical analysis like that arising from MD simulations. Second, the central part of this spectrum is very sensitive to the atom-diatom pair potential, so it constitutes a direct macroscopic manifestation of interactions at the molecular level. ${ }^{8}$ In particular, it has been shown that the central $Q$ branch that the experimental infrared line shapes exhibit is directly related to the particular kind of rotational dynamics of the molecule in the liquid, and thus to the anisotropic interaction potential. ${ }^{9} \mathrm{Fi}-$ nally, there exist adequate forms of the effective potential $\mathrm{HCl}-\mathrm{Ar}$ in the liquid that allow us to reproduce the shape and relative intensities of the branches of the spectrum. ${ }^{6}$ Concretely, experimental $\mathrm{HCl}-\mathrm{Ar}$ line shapes at liquid densities were reproduced even in the case that the anisotropy of the liquid mixture was increased in the experiments by introducing small amounts of $\mathrm{Kr}$ and $\mathrm{Xe} .^{16}$

We performed our simulations on an isotherm, $T_{1}$, and on an isochore, $\rho_{1}$, in the liquid phase of Ar. The objective of such election is to approximately cover all the liquid-phase region of the solvent. The isotherm at $T_{1}=115 \mathrm{~K}$ is composed of four states between near the gas-liquid coexistence line and the liquid-solid line: $\rho_{1}=1.2 \mathrm{~g} / \mathrm{cm}^{3}, \rho_{2}=1.3 \mathrm{~g} / \mathrm{cm}^{3}$, $\rho_{3}=1.4 \mathrm{~g} / \mathrm{cm}^{3}$, and $\rho_{4}=1.5 \mathrm{~g} / \mathrm{cm}^{3}$. The isochore at $\rho_{1}$ $=1.2 \mathrm{~g} / \mathrm{cm}^{3}$ goes from $T_{1}=115 \mathrm{~K}$ to around the critical point temperature for Ar, $T_{4}=160 \mathrm{~K}$. We also considered two temperatures in between: $T_{2}=130 \mathrm{~K}$ and $T_{3}=145 \mathrm{~K}$. Additionally we also simulated conditions at a point on the gas side of the gas-liquid coexistence line on the isotherm $T_{1}=115 \mathrm{~K}$. It corresponds to a density $\rho_{0}=0.045 \mathrm{~g} / \mathrm{cm}^{3}{ }^{17}$

The potential we elect to reproduce $\mathrm{Ar}-\mathrm{Ar}$ interactions is a Lennard-Jones MD standard: $\varepsilon_{\mathrm{Ar}-\mathrm{Ar}}=119.8 \mathrm{~K}$ and $\sigma$ $=3.405 \AA$. Internal vibrations in the $\mathrm{HCl}$ molecule are simulated through a Morse potential $V_{M}=D_{e}\left[1-\exp \left\{\alpha\left(x-x_{e}\right)\right\}\right]^{2}$, where $x=x(t)$ represents the time-dependent $\mathrm{H}-\mathrm{Cl}$ internuclear distance and we take the set of parameters for the isolated molecule proposed by Dijkman and van der Maas: ${ }^{18}$ $x_{e}=1.274 \AA, D_{e}=4.29 \times 10^{4} \mathrm{~cm}^{-1}$, and $\alpha=1.739 \AA^{-1}$. The anisotropic interaction $\mathrm{HCl}-\mathrm{Ar}$ was considered as coming from a site-site potential, SSF2, that was developed and checked in previous works. ${ }^{6}$ It arose as a Lennard-Jones sitesite fit to the Holmgren-Waldman-Klemperer (HWK) II potential proposed several years ago by Holmgren et al. ${ }^{19}$ This potential allows the existence of van der Waals complexes in the gas phase and satisfactorily reproduces the infrared spectra of $\mathrm{HCl}$ solved in liquid Ar or rare-gas liquid mixtures. ${ }^{6}$
The Lennard-Jones parameters of this potential are $\varepsilon_{\mathrm{H}-\mathrm{Ar}}$ $=79.5 \mathrm{~K}, \quad \varepsilon_{\mathrm{Cl}-\mathrm{Ar}}=150.3 \mathrm{~K}, \quad \sigma_{\mathrm{H}-\mathrm{Ar}}=2.41 \AA, \quad$ and $\sigma_{\mathrm{Cl}-\mathrm{Ar}}$ $=3.44 \AA$.

Details on the simulations can be found in Refs. 6 and 9 . For the sake of briefness we only remark that to reproduce highly diluted solutions we consider a single diatomic and $255 \mathrm{Ar}$ atoms. Equations of motion are solved through a leap-frog Verlet algorithm adapted to keep temperature constant. The time step was $0.5 \times 10^{-3} \mathrm{ps}$ in order to properly achieve the vibrational dynamics of the diatomic. After a thermalization process of $50 \mathrm{ps}$ we follow particles dynamics during $6 \times 10^{3} \mathrm{ps}$ in the liquid and $12 \times 10^{3} \mathrm{ps}$ in the gas to obtain adequate statistics. Errors in our objective parameters, that we shall detail in Sec. IV, were estimated by dividing runs in blocks of $1 \times 10^{3} \mathrm{ps}$.

\section{DEFINING WEAKLY BOUND COMPLEXES IN MD SIMULATIONS}

Classical MD simulations consider intermolecular energies arising from continuous interaction potentials and it is not trivial to determine whether the complexation between solute molecules and any solvent atom exists or not. This makes imperative to elect any arbitrary criterion and check its suitability. Apart from having a neat physical meaning, adequate criteria should show settled stability respect to changes in the involved parameters. Typical standards used in the characterization of hydrogen bonds in liquids or other types of dimerization are based upon energetic or geometric supposals. $^{12,13}$

We shall also make use of such criteria in our simulations, but first we discuss a nice idea introduced by Larsen and Stratt $^{20}$ that has been used before in the literature for pure atomic liquids and for diatomic solutes in liquid solvents by our group. At any given time, the mutual-nearestneighbors (mnn) in fluids are defined as such pair of molecules that are close enough to have each other as nearest neighbors. This concept has been successfully applied in order to calculate by means of MD simulations the structural characteristics of weak $\mathrm{HCl}-\mathrm{Ar}$ dimers in liquids. ${ }^{8}$ A definite advantage for its consideration, apart from its simple implementation in simulations, is that it does not need the assumption of any parameter for the system under study. It is only convenient to fix a minimum number of configurations to confirm that a dimer is formed in order to avoid spurious conformations. By taking this limit we get narrower distribution curves of the parameters we are interested in (that we shall detail in the next section), thus improving statistics without going to much longer simulations. We take that time as $5 \mathrm{fs}$, which represents an interval much smaller than that of the rotational relaxation of the diatomic, $\sim 1$ ps. Small variations of this parameter do not affect any of the conclusions of the work. In particular, considering that anisotropic $\mathrm{HCl}-\mathrm{Ar}$ potential surfaces display an absolute minimum at the linear $\mathrm{Ar}-\mathrm{H}-\mathrm{Cl}$ arrangement, ${ }^{14}$ we define a dimer formed under this definition when the $\mathrm{H}$ atom of the diatomic and any Ar atom of the solvent form a mnn for a time longer than 5 fs. One of the main consequences of managing a 
parameter-independent definition is that it allows detailed studies of standard bonding criteria to find out the most adequate intervals for parameters.

Thus, apart from the mnn criterion, we have also elected the geometric and energetic definitions. In the latter we consider that a transient complex is formed when an Ar atom has a potential energy (with respect to its interaction with the molecule) below some value $V_{m}$ and keeps this condition at least for 5 fs. If eventually several atoms would satisfy this condition we take that one with minimum potential energy. For all the liquid conditions considered we choose $V_{m}=$ $-125 \mathrm{~K}$ and for the gas case $V_{m}=-100 \mathrm{~K}$. The deepness of the absolute minimum, $V_{\min }$, of the potential SSF2 is around $-225 \mathrm{~K}$, so our election of $V_{m}$ is between $0.44 V_{\min }$ and $0.55 V_{\min }$. These parameters were elected on the basis of the mnn definition mentioned above. We have checked the stability of this criterion by performing a series of simulations with different values for $V_{m}$ and concluded that neither the evolution with the thermodynamical conditions nor the order of magnitude of the parameters we are interested in change. Only the particular numerical values of such parameters slightly change with $V_{m}$. It should be noted that rigorously in studies where the thermodynamic conditions of the solvent are modified (as our case, because we perform several simulations on an isotherm and an isochore), $V_{m}$ could smoothly change as a consequence of the effective character of interactions in dense phases. However, we have checked that this fact is also irrelevant with respect to the conclusions of this work, so we have fixed the mentioned value for the liquid case for any of the simulated conditions.

The third definition we have adopted is a geometric criterion in which a weak complex turns out when any Ar atom has a distance with respect to the $\mathrm{H}$ atom smaller than $r_{m}$ and forms an angle with the internuclear axis of the diatomic smaller than $\theta_{m}$, where $\theta=0$ corresponds to the linear arrangement $\mathrm{Ar}-\mathrm{H}-\mathrm{Cl}$. Of course, we also add the condition that the dimer is only scored when it satisfies those conditions for at least $5 \mathrm{fs}$. It is important at this moment to remember that experimental measures for the $\mathrm{HCl}-\mathrm{Ar}$ van der Waals complex in the gas phase give $r_{e}=3.98 \AA$ (distance from the center-of-mass of $\mathrm{HCl}$ to the $\mathrm{Ar}$ atom) and $\theta_{e}$ $=41.53^{\circ} .^{21}$ For all the liquid samples we take $r_{m}$ $=4.5 \AA, \theta_{m}=50^{\circ}$ and in the gas case $r_{m}=6.0 \AA, \theta_{m}=120^{\circ}$. We have adopted these sets of parameters after checking that everything in our discussions is affected by small changes in $r_{m}$ or $\theta_{m}$.

\section{LIFETIMES AND PERCENTAGE OF COMPLEXED CONFIGURATIONS}

We have calculated two parameters characterizing the formation dissociation of molecule-atoms pairs: the time that
TABLE I. Residence times (in picosecond) of $\mathrm{HCl}-\mathrm{Ar}$ weak complexes in the liquid along the isochore $\rho_{1}=1.2 \mathrm{~g} / \mathrm{cm}^{3}$ as calculated from different definitions of complexation: mutual-nearest-neighbors ( $\mathrm{mnn})$, energetic criterion (ener), and geometric criterion (geo). Standard deviation uncertainty was obtained by dividing simulations in six steps (see Sec. II for details).

\begin{tabular}{ccccc}
\hline \hline$t_{\text {res }}(\mathrm{ps})$ & $T_{1}=115 \mathrm{~K}$ & $T_{2}=130 \mathrm{~K}$ & $T_{3}=145 \mathrm{~K}$ & $T_{4}=160 \mathrm{~K}$ \\
\hline mnn & $0.106 \pm 0.004$ & $0.103 \pm 0.002$ & $0.097 \pm 0.004$ & $0.094 \pm 0.002$ \\
ener & $0.093 \pm 0.003$ & $0.090 \pm 0.001$ & $0.085 \pm 0.004$ & $0.082 \pm 0.004$ \\
geo & $0.078 \pm 0.003$ & $0.076 \pm 0.002$ & $0.070 \pm 0.003$ & $0.067 \pm 0.002$ \\
\hline \hline
\end{tabular}

certain solvent atom is weakly bound to the molecule, i.e., the residence time, $t_{\text {res }}$, and the fraction of configurations in which any solvent atom is complexed to the molecule. We shall express the last as a percentage and denote it as percentage of configurations ( $\mathrm{pc}$ ). Both calculations were direct in the sense that no correlation function, as sometimes usual in the literature of complexation in dense phases, ${ }^{22,23}$ was involved.

All lifetimes, $t_{\text {res }}$, for liquid conditions are in the scale of subpicoseconds, roughly between 0.07 and 0.1 ps (Tables I and II). Statistical errors are remarkably small, partially as a consequence of fixing a 5 fs limit to consider a dimer is formed. Differences among numerical values arising from the different definitions of dimerization are not significant and the evolution with thermodynamic conditions are similar for the three cases as we shall detail. Geometric definition is the most restrictive, in the sense that it systematically yields to lower values of $t_{\text {res }}$, although standard deviations are similar for all criteria.

It is worthwhile to globally compare the order of magnitude of the results for $t_{\text {res }}$ from the mnn criterion with those obtained by Larsen and Stratt ${ }^{20}$ from the same definition but for the pure liquid Ar. In that case, Ar-Ar residence times obtained with the mnn definition smoothly oscillate from 0.08 ps depending on the particular temperature and density, which constitutes a numerical value slightly smaller (with the mnn definition $t_{\text {res }}$ is in the interval 0.094-0.106 ps) but undoubtedly close to ours. So, there is no evidence after our computations of larger order of magnitude residence times in the case of $\mathrm{HCl}-\mathrm{Ar}$ pairs. Although the deepness of $\mathrm{HCl}-\mathrm{Ar}$ interaction potential $(\sim-225 \mathrm{~K})$ is considerably larger than that of pure $\operatorname{Ar}(\sim-120 \mathrm{~K})$, the energy of the solvent atoms in the liquid is enough to avoid the permanence for a long time of the same Ar atom around the potential well of the molecule.

Evolution of $t_{\text {res }}$ with temperature is similar for all the criteria (see Fig. 1). A decrease with increasing $T$ is observed. The same qualitative dependence was observed for pure Ar (Ref. 20) through MD simulations or simple kinetic

TABLE II. Same that Table I but along the isotherm $T_{1}=115 \mathrm{~K} . \rho_{1}-\rho_{4}$ are four different liquid densities approximately between the gas-liquid $\left(\sim \rho_{1}\right)$ and the liquid-solid $\left(\sim \rho_{4}\right)$ transitions. $\rho_{0}$ is a gas density on the gas side of the gas-liquid coexistence curve at the same temperature.

\begin{tabular}{cccccr}
\hline \hline $\mathrm{t}_{\mathrm{res}}(\mathrm{ps})$ & $\rho_{0}=0.045 \mathrm{~g} / \mathrm{cm}^{3}$ & $\rho_{1}=1.2 \mathrm{~g} / \mathrm{cm}^{3}$ & $\rho_{2}=1.3 \mathrm{~g} / \mathrm{cm}^{3}$ & $\rho_{3}=1.4 \mathrm{~g} / \mathrm{cm}^{3}$ & $\rho_{4}=1.5 \mathrm{~g} / \mathrm{cm}^{3}$ \\
\hline mnn & $0.29 \pm 0.03$ & $0.106 \pm 0.004$ & $0.101 \pm 0.004$ & $0.098 \pm 0.002$ & $0.090 \pm 0.002$ \\
ener & $0.44 \pm 0.09$ & $0.093 \pm 0.003$ & $0.089 \pm 0.003$ & $0.085 \pm 0.001$ & $0.077 \pm 0.005$ \\
geo & $0.28 \pm 0.06$ & $0.078 \pm 0.003$ & $0.076 \pm 0.003$ & $0.074 \pm 0.001$ & $0.070 \pm 0.002$ \\
\hline \hline
\end{tabular}




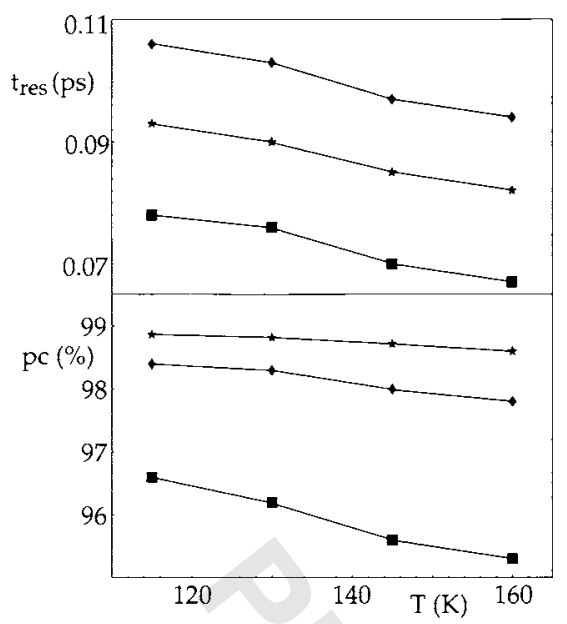

FIG. 1. Evolution with temperature of $t_{\text {res }}$ and $\mathrm{pc}$ for liquid conditions on the isochore $\rho_{1}=1.2 \mathrm{~g} / \mathrm{cm}^{3}$. Diamonds correspond to mutual-nearest-neighbors definition of complexation, stars to the energetic criteria, and squares to the geometric one.

theory arguments. In our case, in a temperature interval of around $45 \mathrm{~K}, t_{\text {res }}$ decreases around $11 \%-15 \%$.

The evolution of $t_{\text {res }}$ with respect to solvent density is displayed in Fig. 2. For the gas density, $\rho_{0}$, residence times are not of different order of magnitude, but between three and five times larger than in the liquid, where times decrease with increasing $\rho$. Evolution is similar for all the criteria, a decrease with increasing density on the isotherm $T_{1}=115 \mathrm{~K}$. Larsen and Stratt $^{20}$ found the same kind of evolution for pure liquid Ar. In the whole liquid region, from the gas-liquid to the vicinity of the liquid-solid transition decrease of $t_{\text {res }}$ amounts $10 \%-20 \%$. Reasoning for simplicity in terms of collisions, more frequent collisions in the liquid and higher

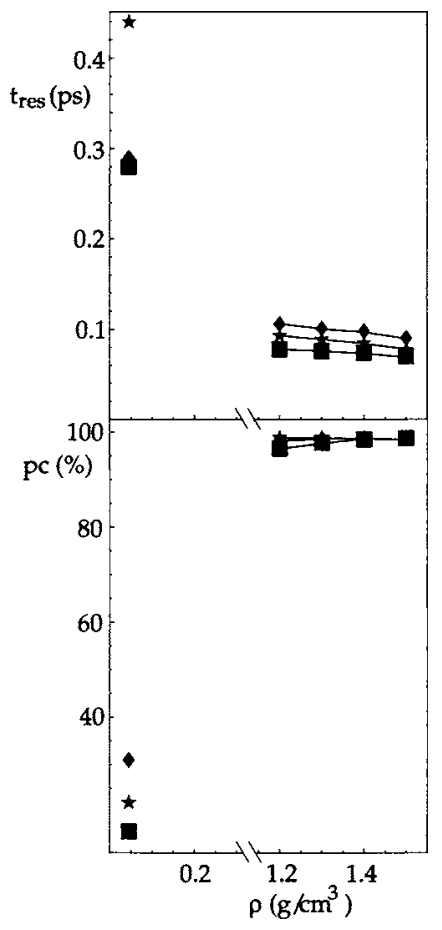

FIG. 2. Evolution with density of $t_{\mathrm{res}}$ and pc on the isotherm $T_{1}=115 \mathrm{~K}$. Note the scale gap between the gas density $\left(\rho_{0}\right)$ (points on the left) and the liquid conditions $\left(\rho_{1}-\rho_{4}\right)$. Symbols are like in Fig. 1.
TABLE III. Percentages of configurations, pc, in which weak dimers in the liquid exist along the isochore $\rho_{1}$. Temperatures are as in Table I.

\begin{tabular}{ccccc}
\hline \hline$p c(\%)$ & $T_{1}$ & $T_{2}$ & $T_{3}$ & $T_{4}$ \\
\hline mnn & $98.4 \pm 0.1$ & $98.3 \pm 0.1$ & $98.0 \pm 0.1$ & $97.8 \pm 0.2$ \\
ener & $98.87 \pm 0.06$ & $98.82 \pm 0.07$ & $98.72 \pm 0.09$ & $98.6 \pm 0.1$ \\
geo & $96.6 \pm 0.3$ & $96.2 \pm 0.1$ & $95.6 \pm 0.2$ & $95.3 \pm 0.2$ \\
\hline \hline
\end{tabular}

solvent-solvent potential energies provoke more rapid formation dissociation of transient complexes. Even at the lowest liquid density the anisotropic interaction of the molecule with the Ar atoms is not capable of retaining solvent atoms close to itself during a long-time interval. On the contrary, in the gas phase, less frequent collisions and less effective solvent-solvent potential energy make Ar atoms stay longer in the region of the molecular potential well.

The pc in which complexation exists (Tables III and IV) is, for the liquid case, always near $100 \%$; i.e., almost at any given instant an Ar atom is associated to the molecule. Although different definitions give different particular values of pc, all the criteria confirm that conclusion. The geometric definition is again the most restrictive. The percentage of complexed configurations decrease when the temperature increases as it would be expected from simple thermal agitation arguments. But it should be noted that the decrease is really small, hardly $0.6 \%-1.3 \%$ in $45 \mathrm{~K}$ temperature interval. Similar to $t_{\text {res }}$ it is unclear from simulations whether the decrease is simply linear or different. With respect to the dependence on density (see Fig. 2), pc increases towards higher densities, which is also easily explainable in a classical picture of finite-time interactions. But actually the increase is relatively small, because in almost any case saturation (existence of complexation in almost all configurations) is reached. These numbers differ from pure liquid Ar, where a value for pc around $50 \%$ was found. ${ }^{20}$ Thus, the attractive form of $\mathrm{HCl}-\mathrm{Ar}$ potential allows very frequent presence of Ar atoms on the vicinity of the potential minimum. Table IV also includes results for gas density $\rho_{0}$. Evidently, there is a considerable skip when passing from the liquid to the gas, where pc is around three to six times smaller, as a consequence of a larger average distance between atoms.

Thus, putting together the results for $t_{\text {res }}$ and $\mathrm{pc}$ in the gas phase, formation of weakly bound dimers is not so frequent, but after formation they last appreciably longer when compared with that in the liquid phases. On the contrary, in the liquid there is no evidence of time stable complexes. Dimers are frequently formed and destroyed, but in such a way that almost at any given time there exists a particular Ar atom weakly complexed to the molecule. Regardless which Ar atom is in the neighborhood of the molecule, there always

TABLE IV. Same that Table III but on the isotherm $T_{1} \cdot \rho_{0}$ corresponds to a gas density at the same temperature. Densities are as in Table II.

\begin{tabular}{cccccc}
\hline \hline$p c(\%)$ & $\rho_{0}$ & $\rho_{1}$ & $\rho_{2}$ & $\rho_{3}$ & $\rho_{4}$ \\
\hline mnn & $31 \pm 6$ & $98.4 \pm 0.1$ & $98.6 \pm 0.2$ & $98.81 \pm 0.08$ & $98.9 \pm 0.1$ \\
ener & $22 \pm 8$ & $98.87 \pm 0.06$ & $98.79 \pm 0.08$ & $98.66 \pm 0.07$ & $98.4 \pm 0.3$ \\
geo & $16 \pm 6$ & $96.6 \pm 0.3$ & $97.8 \pm 0.2$ & $98.5 \pm 0.2$ & $98.73 \pm 0.06$ \\
\hline \hline
\end{tabular}




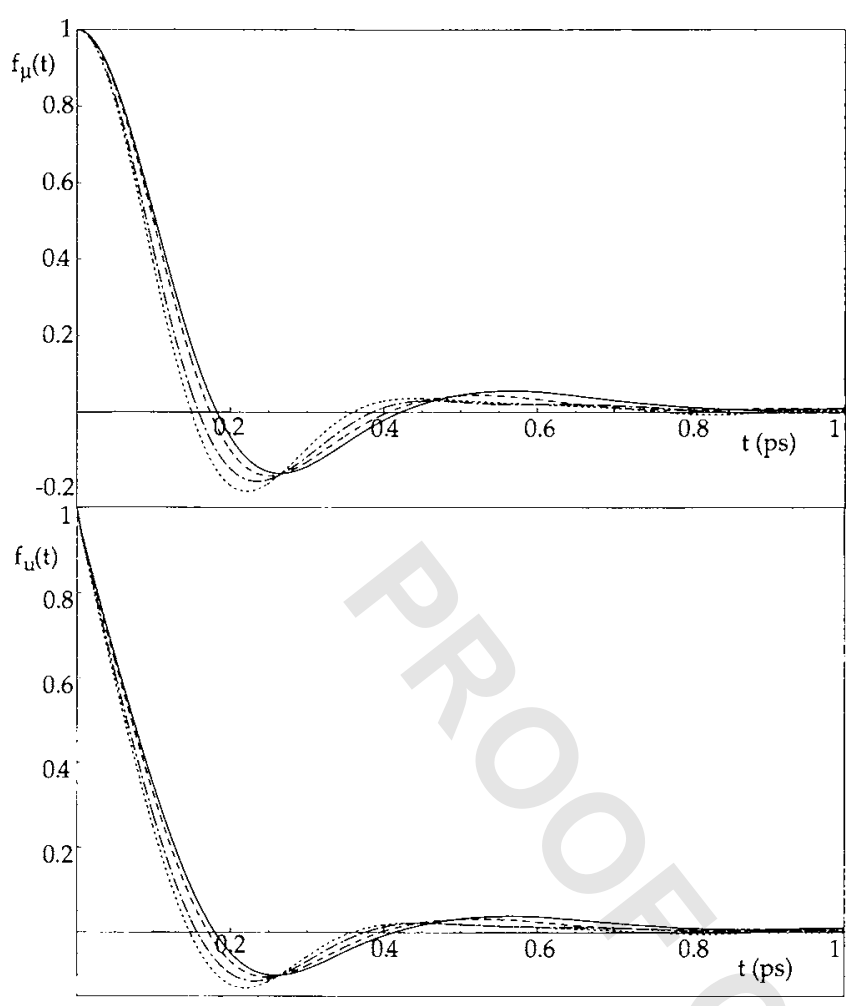

FIG. 3. Time correlation functions, $f_{\mu}(t)$ and $f_{u}(t)$, obtained in the liquid for a fixed density, $\rho_{1}$, and four different temperatures: $T_{1}=115 \mathrm{~K}$ (solid line), $T_{2}=130 \mathrm{~K}$ (dashed), $T_{3}=145 \mathrm{~K}$ (dash-dotted), and $T_{4}=160 \mathrm{~K}$ (dotted). $f_{u}$ has been obtained with the mnn definition of $\mathrm{HCl}-\mathrm{Ar}$ weakly bound dimers.

exists an Ar atom, making the rotational evolution of the molecule very different with respect to the case in which this does not happen. We shall study in the next section precisely how this is manifested on the reorientational dynamics of the molecule, and macroscopically in the infrared spectra of the solution.

\section{ROTATIONAL DYNAMICS OF THE MOLECULAR PROBE}

The rotational dynamics of the diatomic in the solution will be studied through two different time autocorrelation functions (tafs). We shall denote $f_{\mu}(t)$ as the normalized taf of the permanent dipole moment of the molecule, $\boldsymbol{\mu}(t), f_{\mu}(t)=\langle\boldsymbol{\mu}(0) \cdot \boldsymbol{\mu}(t)\rangle /\left\langle\boldsymbol{\mu}(0)^{2}\right\rangle$. This function describes the classical reorientational dynamics of the probe and its Fourier transform is directly measured in spectroscopy experiments yielding to the infrared spectrum of the solution. On the other hand, we have also calculated a function to understand the dynamics of the molecule when a weak $\mathrm{HCl}-\mathrm{Ar}$ dimer is formed. We define the vector, $\mathbf{u}(t)$, as that going from the center of mass of the diatomic to the position of the solvent atom eventually forming the dimer. Then, it is only defined when the transient complex exists in the sense we have discussed in Sec. III. The taf associated to $\mathbf{u}(t)$ will be denoted $f_{u}(t), f_{u}(t)=\langle\mathbf{u}(0)$. $\mathbf{u}(t)\rangle /\left\langle\mathbf{u}(0)^{2}\right\rangle$. In summary, we expect to visualize the rotational evolution of the molecular probe from two viewpoints, by means of $f_{\mu}(t)$ as an effective time function including intervals when dimerization exists or not and by means of $f_{u}(t)$ in time intervals when rotation is influenced by a particular solvent atom.

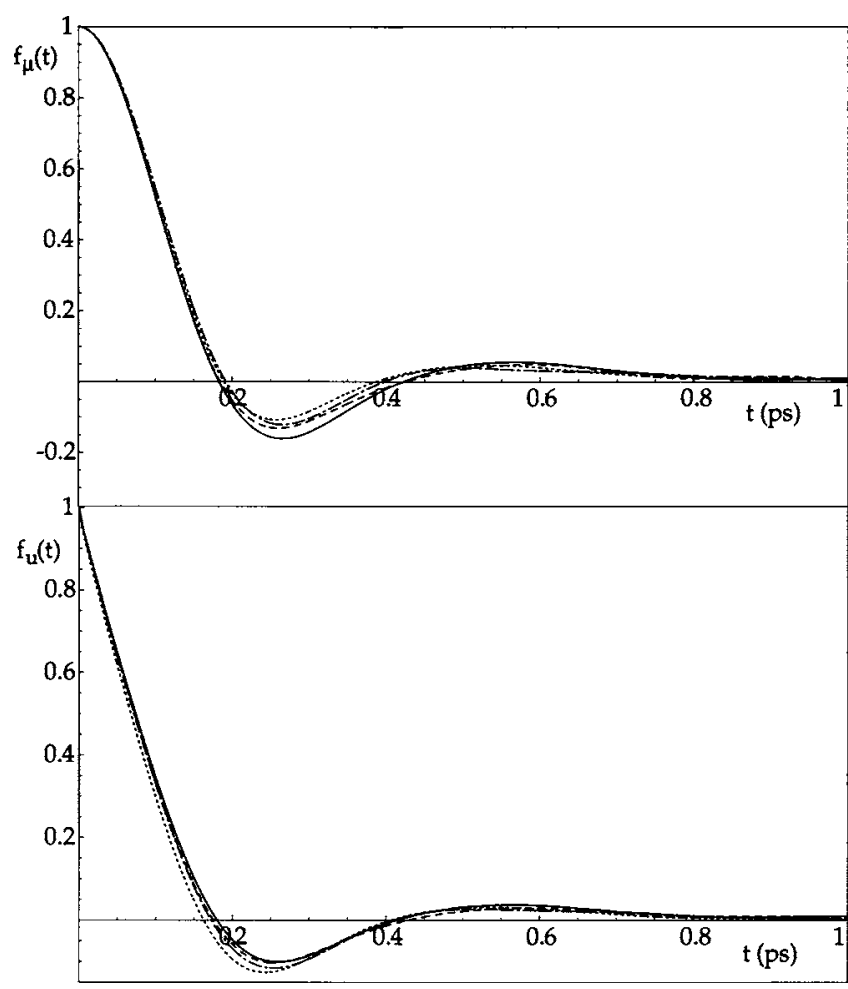

FIG. 4. Time correlation functions, $f_{\mu}(t)$ and $f_{u}(t)$, obtained in the liquid for a fixed temperature, $T_{1}=115 \mathrm{~K}$, and four different liquid densities: $\rho_{1}$ $=1.2 \mathrm{~g} / \mathrm{cm}^{3}$ (thin solid line), $\rho_{2}=1.3 \mathrm{~g} / \mathrm{cm}^{3}$ (dashed), $\rho_{3}=1.4 \mathrm{~g} / \mathrm{cm}^{3}$ (dashdotted), and $\rho_{4}=1.5 \mathrm{~g} / \mathrm{cm}^{3}$ (dotted).

Before the discussion of the results of simulations for such functions we would like to define the correlation times, $t_{\mu}$ or $t_{u}$, as the areas under the corresponding curve; i.e., they are integral correlation times. We take as upper limit for those integrals $0.8 \mathrm{ps}$ in all the liquid phase.

The functions we have obtained for the system under study are depicted in Figs. 3 and 4, and the corresponding integral correlation times in Tables V and VI. In the figures we represent $f_{u}(t)$ obtained with the mnn definition of weakly bound dimer. Functions obtained under the other definitions are very similar. The first important fact is that the global decay of $f_{\mu}(t)$ takes place, for the liquid, in around 1 ps. This means that until decorrelation is complete several transient complexes are formed and erased (we recall that $t_{\text {res }}$ for dimers is always around $0.1 \mathrm{ps}$ ). On the other hand, integral correlation times roughly vary in the liquid from 0.055 to $0.1 \mathrm{ps}$. For all the thermodynamic conditions we consider

TABLE V. Integral correlation times (in picosecond), $t_{\mu}$ and $t_{u}$, of the functions $f_{\mu}(t)$ and $f_{u}(t)$, respectively, calculated as the areas under the functions over the interval $[0,0.8]$ (picosecond). Thermodynamic states correspond to the isochore $\rho_{1}=1.2 \mathrm{~g} / \mathrm{cm}^{3}$ for four different temperatures in the liquid phase.

\begin{tabular}{crrrr}
\hline \hline & $T_{1}$ & $T_{2}$ & $T_{3}$ & $T_{4}$ \\
\hline $\begin{array}{c}t_{\mu}(\mathrm{ps}) \\
t_{u}(\mathrm{ps})\end{array}$ & 0.095 & 0.086 & 0.074 & 0.070 \\
mnn & & & & \\
ener & 0.075 & 0.068 & 0.059 & 0.054 \\
geo & 0.092 & 0.085 & 0.076 & 0.073 \\
\hline \hline
\end{tabular}


TABLE VI. Integral correlation times (in picosecond), $t_{\mu}$ and $t_{u}$, of the functions $f_{\mu}(t)$ and $f_{u}(t)$, respectively, calculated as the areas under the functions over the interval $[0,0.8]$ (picosecond). Thermodynamic states correspond to the isotherm $T_{1}=115 \mathrm{~K}$ for four different densities, $\rho_{1}-\rho_{4}$, in the liquid phase.

\begin{tabular}{crrrr}
\hline \hline & $\rho_{1}$ & $\rho_{2}$ & $\rho_{3}$ & $\rho_{4}$ \\
\hline $\begin{array}{c}t_{\mu}(\mathrm{ps}) \\
t_{u}(\mathrm{ps}) \\
\text { mnn }\end{array}$ & 0.093 & 0.096 & 0.102 & 0.104 \\
ener & 0.074 & 0.069 & 0.067 & 0.061 \\
geo & 0.090 & 0.089 & 0.087 & 0.087 \\
\hline \hline
\end{tabular}

$t_{\mu}$ and $t_{u}$ are similar, although in general $t_{u}$ is slightly smaller than $t_{\mu}$. With respect to the evolution with temperature at a fixed density, behavior is clear (see Table V): a decrease with increasing temperature is observed both for $t_{\mu}$ and $t_{u}$. The interpretation of this conduct is direct, because the thermal agitation tends to accelerate the orientational decorrelation in any case, whether a dimer is formed or not. Dependence with density (Table VI) is more subtle, because $t_{\mu}$ appears to slightly increase with density but the evolution for $t_{u}$ is not so evident. Depending on the elected definition a weak decrease is observed with increasing density or even an almost constant value.

The qualitative time evolution of $f_{\mu}(t)$ and $f_{u}(t)$ is very interesting (see Figs. 3 and 4). The first intriguing fact is that for any liquid condition both functions evolve in a very similar way. This means that in the liquid globally reorientation of the diatomic is associated to weak dimerization; in other words, the dimer $\mathrm{HCl}-\mathrm{Ar}$ effectively rotates as a whole. This is also what one should expect from the results for $t_{\text {res }}$ and pc commented in the preceding section. Although the residence time each Ar atom is associated to the molecule is not long, the formation-dissociation rate is so high that in effective terms there is always an Ar atom influencing the reorientational dynamics of the diatomic. In this sense, we could talk of a peculiar transient complexation not long-time stable but with consequences with respect to the reorientation of the molecular probe as dimer existence was much longer in time. This rotational complex is not only associated to a particular Ar atom but several different atoms influence the dynamics of the diatomic molecule.

Time evolution of these tafs in the liquid can be separated in four regions: a first rapid decay up to approximately $0.2 \mathrm{ps}$, a negative dip between 0.2 and 0.4 ps reaching negative values in the interval $[-0.15,-0.20]$, a positive lowamplitude bounce between 0.4 and 0.8 ps, and a final positive decay which is completed at around 1 ps. Previous works showed that this particular reorientational dynamics could be described as a hindered rotation. ${ }^{9}$ This is the rotational evolution of a species with larger moment of inertia than the diatomic itself, performing its orientational evolution in the cage formed by the closest solvent atoms. The macroscopic reflect of this peculiar reorientational dynamics is the shape of the infrared spectra of the system in the frequency region between the maxima of $P$ and $R$ branches. In this region absorption is in principle forbidden due to selection rules, but hindered rotation leads to the appearance of an

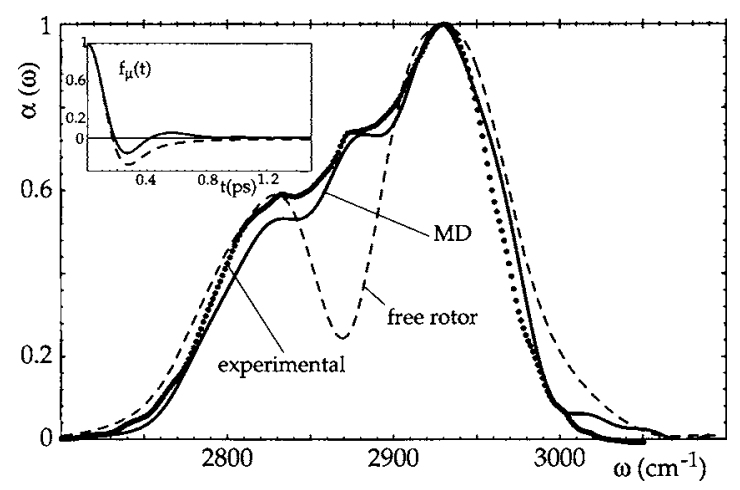

FIG. 5. Experimental infrared spectrum (dots) of $\mathrm{HCl}$ solved in liquid $\mathrm{Ar}$ at $T_{1}=115 \mathrm{~K}$ and $\rho_{1}=1.2 \mathrm{~g} / \mathrm{cm}^{3}$, theoretical spectrum obtained in the free rigid-rotor limit (dashed), and simulated spectra (solid line). The inset displays the corresponding $f_{\mu}(t)$ : free rigid rotor (dashed) and simulated hindered rotor (solid).

intense absorption (sometimes comparable with the intensity of the allowed $P$ and $R$ branches) known as $Q$-branch absorption. ${ }^{9}$ At this point, a direct comparison of the rotational evolution revealed by $f_{\mu}(t)$ with its macroscopic spectral manifestation maybe elucidating. We have compiled in Fig. 5 some previous results ${ }^{8}$ that can help us to understand the importance of hindered rotation behavior in opposition to the free rigid-rotor case. The figure shows the experimental absorption spectrum of $\mathrm{HCl}-\mathrm{Ar}$ at $T_{1}=115 \mathrm{~K}$ and $\rho_{1}$ $=1.2 \mathrm{~g} / \mathrm{cm}^{3}$ and those line shapes obtained by Fourier transforming $f_{\mu}(t)$ obtained from MD simulations and the theoretical $f_{\mu}(t)$ arising from the free rigid-rotor limit. ${ }^{24}$ The latter exhibits a deeper negative minimum $(\sim-0.25)$ and hereafter a monotonic run up towards decorrelation through negative values. This rotational dynamics is not capable of reproducing the experimental absorption band. It does not yield to a $Q$ branch in the $P-R$ interbranch region, that, on the contrary, MD simulations satisfactorily reproduce. Moreover, the external decay of the $P-R$ rotational wings is only accomplished through MD simulations. Free rigid-rotor limit gives slower decays that produce a too broad band.

Evolution with temperature of simulated $f_{\mu}(t)$ and $f_{u}(t)$ is similar (see Fig. 3). As temperature increases, hindering of reorientational evolution decreases. This is reflected as a deeper negative dip and a smaller positive rebound as $T$ increases. In terms of the parameters $t_{\text {res }}$ and pc, this is easy to understand, because both decrease with temperature, making the consequences of dimerization smaller. This evolution explains the experimentally observed behavior of the relative intensity of the $Q$ branch in the spectral profiles: it decreases with increasing temperature. ${ }^{4,25,26}$

Nevertheless, the evolution of the qualitative behavior of $f_{\mu}(t)$ and $f_{u}(t)$ with density at a fixed temperature is more subtle (see Fig. 4). This is because of two main facts. First, the evolution of $t_{\text {res }}$ and pc with density is opposite (compare both pictures of Fig. 2) making unclear the global behavior of complexation with density. And second, previous MD calculations $^{8}$ of the radial-orientational $\mathrm{HCl}-\mathrm{Ar}$ pair distribution function, $g(r, \theta)$, showed that the hindering of rotational evolution is greatly influenced by the relative size of $\mathrm{HCl}-\mathrm{Ar}$ dimers with respect to the size and shape of the first solva- 
tion shell around the diatomic probe, which strongly depends on solvent density. So, the combined effect of dimerization and the room dimers having to rotate determines the behavior of $f_{\mu}(t)$ and $f_{u}(t)$ with density. Unfortunately, experimental studies ${ }^{4,25}$ of the evolution of spectral line shapes with density at a fixed temperature are scarce and limited to narrow density intervals, making it difficult to evaluate the evolution of the relative intensity of the $Q$ branch with solvent density.

\section{CONCLUSIONS}

Classical molecular-dynamics simulations have allowed us to study the formation dissociation of $\mathrm{HCl}-\mathrm{Ar}$ weakly bound pairs in the liquid phase through the consideration of an anisotropic diatom-atom potential that in the low-density limit reproduces van der Waals complexation. We adopted three different definitions of dimerization. First, a parameterindependent one in which a dimer is formed when the $\mathrm{H}$ atom of the molecule and a close solvent Ar atom form a mutual-nearest-neighbor pair. Second, an energetic characterization taking an upper limit, $V_{m}$, for the $\mathrm{Ar}-\mathrm{HCl}$ pair potential energy, and third a geometric one depending only on a distance, $r_{m}$, and on an angle, $\theta_{m}$. All these definitions yield to similar values for the lifetimes, $t_{\text {res }}$, and the percentage of configurations in which complexation exists, pc. Lifetimes are always in a subpicosecond time scale, but interestingly, pc always almost reaches $100 \%$ in liquid conditions. Evolution of these parameters with temperature at a fixed density or with density on an isotherm are similar with the three definitions. Complexation effects clearly diminish with increasing temperature, but behavior with density is more complicated, because $t_{\text {res }}$ decreases with increasing density but the evolution of pc is contrary. Nevertheless, at gas densities lifetimes are between three and five times larger than in the liquid and pc decreases to values of around 15\%-30\%.

We have also computed two time autocorrelation functions, $f_{\mu}(t)$ and $f_{u}(t)$, that allows us to study the rotational evolution of the molecular probe whether transient complexes are formed or not. From their comparison we conclude that at liquid conditions reorientation is directly associated to weak complexation. This provokes a peculiar hindered rotational dynamics.

In conclusion we would like to stress that in the liquid phase, anisotropic $\mathrm{HCl}-\mathrm{Ar}$ potential that in the low-density limit reproduces van der Waals complexation gives rise to the unstable dimers (with subpicosecond lifetimes). They are very frequently formed and broken down in such a rapid way that in almost each time step there exists an Ar atom striking the reorientational dynamics of the diatomic probe. Although different Ar atoms are involved in such complexation process, the effect on reorientational dynamics is similar to the existence of a fixed one. Thus, weakly bound dimerization hinders the reorientational motion of the diatomic provoking peculiar time autocorrelation functions for its dipole moment and characteristic infrared line shapes. This is a feasible ex- planation for the observed third peak, the so-called $Q$ branch, in the forbidden interbranch region between the $P$ and $R$ branches that experimental absorption coefficients display for this system. Although we have elected as prototypical system with an interesting anisotropic potential the pair $\mathrm{HCl}-\mathrm{Ar}$, this could be extended to other solute-solvent pairs interacting via strongly anisotropic potential surfaces.

\section{ACKNOWLEDGMENTS}

We thank financial support from Ministerio de Ciencia y Tecnología of Spain under Grant No. BFM2002-01225 FEDER, BFM2003-07106, and FIS2005-05081. We also acknowledge support by Junta de Castilla y León of Spain under Grant No. SA080/04 and SA092/04.

${ }^{1}$ M. O. Bulanin and N. D. Orlova, Opt. Spectrosc. 4, 569 (1958); J. Lascombe, P. V. Huong, and M. L. Jossien, Bull. Soc. Chim. Fr. 7-8, 1175 (1959)

${ }^{2}$ J. P. Marsault, F. Marsault-Herail, and G. Lévi, J. Chem. Phys. 62, 893 (1975).

${ }^{3}$ N. N. Filippov and K. S. Rutkowski, J. Mol. Liq. 92, 251 (2001).

${ }^{4}$ For a review see, M. O. Bulanin, S. Velasco, and A. Calvo Hernández, J. Mol. Liq. 70, 107 (1996).

${ }^{5}$ K. G. Tokhadze and Z. Mielke, J. Chem. Phys. 99, 5071 (1993); K. G. Tokhadze, S. S. Utkina, N. N. Filippov, and Z. Mielke, Opt. Spectrosc. 79, 536 (1995).

${ }^{6}$ A. Medina, J. M. M. Roco, A. Calvo Hernández, S. Velasco, M. O. Bulanin, W. A. Herrebout, and B. J. van der Veken, J. Chem. Phys. 116, 5058 (2002).

${ }^{7}$ For a review see, Structure and Dynamics of Weakly-Bound Complexes, edited by A. Weber (Reidel, Dordrecht, 1987).

${ }^{8}$ A. Medina, J. M. M. Roco, A. Calvo Hernández, and S. Velasco, J. Chem. Phys. 119, 5176 (2003).

${ }^{9}$ A. Medina, J. M. M. Roco, A. Calvo Hernández, and S. Velasco, J. Chem. Phys. 121, 6353 (2004).

${ }^{10}$ See Figs. 2 and 3 in Ref. 9.

${ }^{11}$ For a review see, A. I. Burshtein and S. I. Temkin, Spectroscopy of Molecular Rotation in Gases and Liquids (Cambridge University Press, Cambridge, 1994).

${ }^{12}$ D. Bertolini, M. Cassettari, M. Ferrario, P. Grigolini, and G. Salvetti, Adv. Chem. Phys. 62, 277 (1985).

${ }^{13}$ A. Geiger and P. Mausbach, in Hydrogen Bonded Liquids, NATO ASI Series C, Vol. 329, edited by J. C. Dore and J. Teixeira (Kluwer, The Netherlands, 1991), pp. 181-183 and references therein.

${ }^{14}$ G. C. Maitland, M. Rigby, E. B. Smith, and W. A. Wakeham, Intermolecular Forces: Their Origin and Determination, (Clarendon, Oxford, 1981), pp. 426-428.

${ }^{15}$ J. Pérez, D. Luis, A. Calvo Hernández, and S. Velasco, J. Chem. Phys. 91, 3443 (1989).

${ }^{16}$ See Figs. 1 and 2 in Ref. 6.

${ }^{17}$ V. A. Rabinovich, V. I. Nedostup, A. A. Vasserman, and L. S. Veksler, Thermophysical Properties of Neon, Argon, Krypton, and Xenon (Hemisphere, Washington, 1988).

${ }^{18}$ F. G. Dijkman and J. H. van der Maas, J. Chem. Phys. 66, 3871 (1977).

${ }^{19}$ S. L. Holmgren, M. Waldman, and W. Klemperer, J. Chem. Phys. 69, 1661 (1978).

${ }^{20}$ R. E. Larsen and R. M. Stratt, Chem. Phys. Lett. 297, 211 (1998).

${ }^{21}$ G. T. Fraser and A. S. Pine, J. Chem. Phys. 85, 2502 (1986).

${ }^{22}$ M. Matsumoto and K. E. Gubbins, J. Chem. Phys. 93, 1981 (1990).

${ }^{23}$ E. Guàrdia, J. Martí, J. A. Padró, L. Saiz, and A. V. Komolkin, J. Mol. Liq. 96, 3 (2002).

${ }^{24}$ B. Guillot, Ph. Marteau, and J. Obriot, Mol. Phys. 65, 765 (1988).

${ }^{25}$ M. O. Bulanin, K. Kerl, A. Padilla, A. Calvo Hernández, and J. Pérez, Phys. Chem. Chem. Phys. 5, 285 (2003).

${ }^{26}$ J. Pérez, A. Padilla, W. A. Herrebout, B. J. van der Veken, A. Calvo Hernández, and M. O. Bulanin, J. Chem. Phys. 122, 194507 (2005). 